\title{
Generalized matrix models and AGT correspondence at all genera
}

\author{
Giulio Bonelli, ${ }^{a}$ Kazunobu Maruyoshi, ${ }^{a}$ Alessandro Tanzini ${ }^{a}$ and Futoshi Yagi $^{b}$ \\ ${ }^{a}$ International School of Advanced Studies (SISSA) and INFN, Sezione di Trieste, \\ via Bonomea 265, 34136 Trieste, Italy \\ ${ }^{b}$ Institut des Hautes Études Scientifiques, \\ 91440, Bures-sur-Yvette, France \\ E-mail: bonelli@sissa.it, maruyosh@sissa.it, tanzini@sissa.it, \\ fyagi@ihes.fr
}

\begin{abstract}
We study generalized matrix models corresponding to $n$-point Virasoro conformal blocks on Riemann surfaces with arbitrary genus $g$. Upon AGT correspondence, these describe four dimensional $\mathcal{N}=2 \mathrm{SU}(2)^{n+3 g-3}$ gauge theories with generalized quiver diagrams. We obtain the generalized matrix models from the perturbative evaluation of the Liouville correlation functions and verify the consistency of the description with respect to degenerations of the Riemann surface. Moreover, we derive the Seiberg-Witten curve for the $\mathcal{N}=2$ gauge theory as the spectral curve of the generalized matrix model, thus providing a check of AGT correspondence at all genera.
\end{abstract}

KeYwords: Supersymmetric gauge theory, Matrix Models, Extended Supersymmetry, Conformal and W Symmetry 


\section{Contents}

1 Introduction 1

2 From Liouville theory to generalized matrix model 2

3 Degenerations $\quad 6$

$\begin{array}{lll}3.1 & \text { Degenerations of punctured tori } & 7\end{array}$

4 Spectral curve of the generalized matrix model $\quad 9$

$\begin{array}{ll}4.1 \text { Dependence on internal momenta } & 13\end{array}$

5 Conclusions $\quad 16$

$\begin{array}{ll}\text { A Addendum: playing with genus } 2 \text { curves } & 17\end{array}$

\section{Introduction}

The distinctive feature of M-theory is the description in geometrical terms of nonperturbative phases of superstrings. This approach is very effective for local geometries, where the dynamics of gravitational degrees of freedom gets decoupled and we gain a framework for the description of non-perturbative gauge theory dynamics. M-theory beautifully encodes the Seiberg-Witten geometry of four dimensional $\mathcal{N}=2$ theories in terms of M5-brane compactifications [1-3]. In particular in [3] a full class of generalized quiver gauge theories has been described in terms of multiple M5-brane systems covering a generic punctured Riemann surface $\mathcal{C}_{g, n}$. For example, for $\mathcal{C}_{0, n}$ and $\mathcal{C}_{1, n}$ one recovers Witten's constructions of linear and circular quivers in the appropriate degeneration limits.

In this context a very intriguing relation between the partition function of four dimensional $\mathrm{SU}(2)^{n+3 g-3}$ superconformal $\mathcal{N}=2$ gauge theories [4] and Liouville theory on $\mathcal{C}_{g, n}$ has been discovered in [5]. This proposal has been a subject of intensive investigations and refinements from different viewpoints. Evidence for this conjecture as well as complete proofs for some cases can be found in [6-18]. Extensions to higher rank gauge groups and Toda field theories were introduced and discussed in [19-25]. The refinement of the correspondence in presence of gauge theory observables has been presented and studied in [26-42]. Moreover, some arguments for the derivation of the AGT correspondence were proposed in the M-theory context in $[43,44]$ and via matrix models in [45-66].

Here we would like to address this correspondence from a complementary point of view, explaining how to recover the geometry of the M-theory set-up and the Seiberg-Witten data in the wildest generality. To this end we derive a generalized matrix model from Liouville theory on $\mathcal{C}_{g, n}$ and study its large $N$ limit recovering the gauge theory Seiberg-Witten curve as its spectral curve. This provides a check of the AGT conjecture at all genera. 
In section 2 we derive the generalized matrix model — as extended Selberg integrals starting from the Coulomb gas representation of the residues of the perturbative Liouville theory correlators. The matrix model potential that we get has the form anticipated by [45] and in the elliptic case it coincides with the one derived in [64].

In section 3 we discuss the stability of this picture and its consistency with respect to the degeneration of the curve $\mathcal{C}_{g, n}$ in general and present the degenerations of punctured tori as an explicative example.

In section 4 we analyze the large $N$ limit and show how, by consistently adapting to our case the standard matrix model techniques, one gets a spectral curve in terms of quadratic differentials on $\mathcal{C}_{g, n}$ precisely reproducing the Seiberg-Witten curve and differential proposed in [3].

We leave our concluding remarks to section 5 and devote an appendix to the detailed study of the degenerations of the $\mathcal{C}_{2,0}$ Seiberg-Witten data.

\section{From Liouville theory to generalized matrix model}

In this section we derive the generalized matrix model which corresponds to the $n$ point conformal block on a Riemann surface $\mathcal{C}_{g}$ of genus $g$. We derive it from the perturbative calculation of the correlation function of the Liouville theory by following the discussion in [67].

The $n$-point function of the Liouville theory on $\mathcal{C}_{g}$ is given by the following path integral

$$
A \equiv\left\langle\prod_{k=1}^{n} e^{-2 m_{k} \phi\left(w_{k}, \bar{w}_{k}\right)}\right\rangle_{\text {Liouville on } \mathcal{C}_{g}} \equiv \int \mathcal{D} \phi(z, \bar{z}) e^{-S[\phi]} \prod_{k=1}^{n} e^{-2 m_{k} \phi\left(w_{k}, \bar{w}_{k}\right)},
$$

where the Liouville action is given by

$$
S[\phi]=\frac{1}{4 \pi} \int d^{2} z \sqrt{g}\left(g^{a b} \partial_{a} \phi \partial_{b} \phi+Q R \phi+4 \pi \mu e^{2 b \phi}\right) .
$$

We divide the Liouville field into the zero mode and the fluctuation $\phi(z, \bar{z})=\phi_{0}+\tilde{\phi}(z, \bar{z})$, obtaining

$$
A=\int \mathcal{D} \tilde{\phi} e^{-\tilde{S}} \prod_{k=1}^{n} e^{-2 m_{k} \tilde{\phi}\left(w_{k}, \bar{w}_{k}\right)} \int_{-\infty}^{+\infty} d \phi_{0} e^{-\mu e^{2 b \phi_{0}} \int d^{2} z \sqrt{g} e^{2 b \tilde{\phi}}} e^{-\frac{Q \phi_{0}}{4 \pi} \int d^{2} z \sqrt{g} R} e^{-2 \phi_{0} \sum_{k} m_{k}},
$$

where

$$
\tilde{S}=\frac{1}{4 \pi} \int d^{2} z \sqrt{g}\left(g^{a b} \partial_{a} \tilde{\phi} \partial_{b} \tilde{\phi}+Q R \tilde{\phi}\right) .
$$

We can integrate out the zero mode $\phi_{0}$ as

$$
\int_{-\infty}^{+\infty} d \phi_{0} e^{-\mu e^{2 b \phi_{0}} \int d^{2} z \sqrt{g} e^{2 b \tilde{\phi}}} e^{-2(g-1) Q \phi_{0}} e^{-2 \phi_{0} \sum_{k} m_{k}}=\frac{\mu^{N} \Gamma(-N)}{2 b}\left(\int d^{2} z \sqrt{g} e^{2 b \tilde{\phi}}\right)^{N},
$$

where we have used $\int d^{2} z \sqrt{g} R=4 \pi \chi=8 \pi(1-g)$ and $N$ is defined as

$$
N \equiv \frac{1}{b} \sum_{k} m_{k}+\frac{Q}{b}(1-g) \text {. }
$$


Therefore, the $n$-point function can be written as

$$
A=\frac{\mu^{N} \Gamma(-N)}{2 b} \int \mathcal{D} \tilde{\phi} e^{-\tilde{S}}\left(\int d^{2} z \sqrt{g} e^{2 b \tilde{\phi}}\right)^{N} \prod_{k=1}^{n} e^{-2 m_{k} \tilde{\phi}\left(w_{k}, \bar{w}_{k}\right)} .
$$

When $N \in \mathbb{Z}_{\geq 0}$, the correlator diverges due to the factor $\Gamma(-N)$. The residues $A_{N}$ at these simple poles are computed then in perturbation theory in $b$ around the free scalar field action (2.4). From now on, our convention is that

$$
\langle\ldots\rangle_{\text {free on } \mathcal{C}_{g}}=\int \mathcal{D} \tilde{\phi} e^{-\frac{1}{4 \pi} \int d^{2} z \sqrt{g} g^{a b} \partial_{a} \tilde{\phi} \partial_{b} \tilde{\phi}} \ldots
$$

which leads to

$$
A_{N}=\frac{(-\mu)^{N}}{2 b N !}\left\langle e^{-\frac{Q}{4 \pi} \int d^{2} z \sqrt{g} R \tilde{\phi}(z)} \prod_{i=1}^{N} \int d^{2} z_{i} \sqrt{g} e^{2 b \tilde{\phi}\left(z_{i}\right)} \prod_{k=1}^{n} e^{-2 m_{k} \tilde{\phi}\left(w_{k}\right)}\right\rangle_{\text {free on } \mathcal{C}_{g}}
$$

The condition (2.6) ensures momentum conservation in the free theory.

Here we choose as a reference volume form $d^{2} z \sqrt{g}=|\omega(z) d z|^{2}$ where $\omega(z)$ is the coefficient of a reference holomorphic differential. This differential has $2 g-2$ zeros, which we denote by $\xi_{I}(I=1, \cdots 2 g-2)$. Then, the first factor in the expectation value of (2.9) becomes

$$
\frac{Q}{2 \pi} \int d^{2} z \tilde{\phi}(z) \partial \bar{\partial} \log |\omega|^{2}=\frac{Q}{2 \pi} \int d^{2} z \tilde{\phi}(z) \sum_{I=1}^{2 g-2}(2 \pi) \delta^{2}\left(z-\xi_{I}\right)=Q \sum_{I=1}^{2 g-2} \tilde{\phi}\left(\xi_{I}\right),
$$

where we have used $R=-(2 / \sqrt{g}) \partial \bar{\partial} \log \sqrt{g}$. Thus, we obtain

$$
A_{N}=\frac{(-\mu)^{N}}{2 b N !}\left\langle\prod_{I=1}^{2 g-2} e^{Q \tilde{\phi}\left(\xi_{I}\right)} \int \prod_{i=1}^{N} d^{2} z_{i}\left|\omega\left(z_{i}\right)\right|^{2} e^{2 b \tilde{\phi}\left(z_{i}\right)} \prod_{k=1}^{n} e^{-2 m_{k} \tilde{\phi}\left(w_{k}\right)}\right\rangle_{\text {free on } \mathcal{C}_{g}} .
$$

The $\ell$-point function of the free theory on $\mathcal{C}_{g}$ is given in the factorized form as [68-70]

$$
\begin{aligned}
& \left\langle\prod_{i=1}^{\ell} e^{i k_{i} \phi\left(z_{i}, \bar{z}_{i}\right)}\right\rangle_{\text {free on } \mathcal{C}}=(\operatorname{det} \operatorname{Im} \tau)^{1 / 2} \delta\left(\sum_{i} k_{i}\right) \times \\
& \int_{-\infty}^{\infty} \prod_{a=1}^{g} d p_{a}\left|\prod_{i=1}^{\ell} \omega\left(z_{i}\right)^{-k_{i}^{2} / 4} \prod_{i<j} E\left(z_{i}, z_{j}\right)^{k_{i} k_{j} / 2} \exp \left(2 \pi i \sum_{a, b} p_{a} p_{b} \tau_{a b}+2 \pi i \sum_{a, i} p_{a} k_{i} \int^{z_{i}} \omega_{a}\right)\right|^{2}
\end{aligned}
$$

where $\tau_{a b}$ is the period matrix, $E\left(z_{i}, z_{j}\right)$ is the prime form, $\left\{\omega_{a}\right\}$ is a basis of normalized holomorphic one-forms, and $p_{a}$ is interpreted as the momentum flowing through the $a$-th A-cycle. 
Using the explicit expression (2.12) for (2.9), we find that the residue $A_{N}$ of the $n$-point function of the Liouville theory reduces to the following integral

$$
\begin{aligned}
A_{N} \propto & \prod_{a=1}^{g} \int_{-\infty}^{+\infty} d p_{a}\left|C(w, m, \xi, p) \exp \left(2 \pi i \sum_{a, b} p_{a} p_{b} \tau_{a b}\right)\right|^{2} \\
& \prod_{i=1}^{N} \int d^{2} z_{i}\left|\omega\left(z_{i}\right)\right|^{2+2 b^{2}}\left|\exp \left(4 \pi b \sum_{a, i} p_{a} \int^{z_{i}} \omega_{a}\right)\right|^{2} \\
& \left|\prod_{i<j} E\left(z_{i}, z_{j}\right)^{-2 b^{2}} \prod_{i, k} E\left(z_{i}, w_{k}\right)^{2 b m_{k}} \prod_{I, i} E\left(\xi_{I}, z_{i}\right)^{-1-b^{2}}\right|^{2},
\end{aligned}
$$

where the factor $C(w, m, \xi, p)$ was defined as

$$
\begin{aligned}
C(w, m, \xi, p)= & \prod_{I} \omega\left(\xi_{I}\right)^{\frac{Q^{2}}{4}} \prod_{k=1}^{n} \omega\left(w_{k}\right)^{\frac{m_{k}^{2}}{g_{s}^{2}}} \prod_{k<\ell} E\left(w_{k}, w_{\ell}\right)^{-\frac{2 m_{k} m_{\ell}}{g_{s}^{2}}} \prod_{I, k} E\left(\xi_{I}, w_{k}\right)^{\frac{Q m_{k}}{g_{s}}} \\
& \times \prod_{I<J} E\left(\xi_{I}, \xi_{J}\right)^{-\frac{Q^{2}}{2}} \exp \left[2 \pi \sum_{a} p_{a}\left(Q \sum_{I} \int^{\xi_{I}} \omega_{a}-2 \sum_{k} m_{k} \int^{w_{k}} \omega_{a}\right)\right] .
\end{aligned}
$$

As in the torus case [64], it is not straightforward to factorize the integrals over the Riemann surface into holomorphic and anti-holomorphic integrals for generic $N$. However this is easily performed in the large $N$ limit. Indeed, the last two-lines of (2.13) can be written as

$$
\int \prod_{i} d^{2} z_{i}\left|\mu \mathrm{e}^{\frac{b}{g_{s}} W}\right|^{2} \sim\left|\int \prod_{i} d z_{i} \mu \mathrm{e}^{\frac{b}{g_{s}} W}\right|^{2}
$$

where $\mu$ and $W$ are

$$
\begin{aligned}
\mu & =\left[\omega\left(z_{i}\right)^{1+b^{2}} \prod_{i, I} E\left(z_{i}, \xi_{I}\right)^{-1-b^{2}}\right] \prod_{1 \leq i<j \leq N} E\left(z_{i}, z_{j}\right)^{-2 b^{2}} \prod_{i} E\left(z_{i}, z^{*}\right)^{2 b \sum_{k} m_{k} / g_{s}} \\
W & =\sum_{i=1}^{N}\left(\sum_{k=1}^{n} 2 m_{k} \log \frac{E\left(z_{i}, w_{k}\right)}{E\left(z_{i}, z^{*}\right)}+4 \pi \sum_{a=1}^{g} p_{a} \int^{z_{i}} \omega_{a}\right)
\end{aligned}
$$

where we have chosen a base point $z^{*}$ in order to split the measure from the potential and we have rescaled the parameters as $m_{k} \rightarrow m_{k} / g_{s}$ and $p_{a} \rightarrow p_{a} / g_{s}$. Notice that the term in the square brackets in (2.16) is independent on the zeroes of the conformal factor, ensuring therefore that the generalized matrix model correctly embodies the conformal symmetry of Liouville theory.

The large $N$ limit amounts to take $g_{s} \rightarrow 0$ keeping $g_{s} N, b, m_{k}$ and $p_{a}$ finite. In this limit, the conditions for the criticality are given by

$$
b \sum_{j \neq i} \frac{E^{\prime}\left(z_{i}, z_{j}\right)}{E\left(z_{i}, z_{j}\right)} d z_{i}-\sum_{k=1}^{n} m_{k} \frac{E^{\prime}\left(z_{i}, w_{k}\right)}{E\left(z_{i}, w_{k}\right)} d z_{i}-2 \pi \sum_{a=1}^{g} p_{a} \omega_{a}\left(z_{i}\right)=0
$$

where $E^{\prime}\left(z_{1}, z_{2}\right) \equiv \partial_{z_{1}} E\left(z_{1}, z_{2}\right)$. The conditions obtained from the $\bar{z}_{i}$-derivatives are just the complex conjugate of (2.18). It is remarkable that the conditions for criticality are separated 
into holomorphic and anti-holomorphic equations, which implies that the integrals over the Riemann surface in (2.13) can be factorized into holomorphic and anti-holomorphic integrals in the large $N$ limit as stated in (2.15). We are therefore left with the following matrix-like integral

$$
\begin{aligned}
& Z_{N}^{\mathcal{C}_{g, n}}(\mathrm{w}, \mathrm{m}, \mathrm{p}, \mathrm{v}) \equiv \\
& \quad \int \prod_{i=1}^{N} d z_{i}\left[\omega\left(z_{i}\right)^{1+b^{2}} \prod_{i, I} E\left(z_{i}, \xi_{I}\right)^{-1-b^{2}}\right] \prod_{1 \leq i<j \leq N} E\left(z_{i}, z_{j}\right)^{-2 b^{2}} \prod_{i} E\left(z_{i}, z^{*}\right)^{2 b \sum_{k} m_{k} / g_{s}} \\
& \quad \times \exp \left(\frac{b}{g_{s}} \sum_{i=1}^{N}\left(\sum_{k=1}^{n} 2 m_{k} \log \frac{E\left(z_{i}, w_{k}\right)}{E\left(z_{i}, z^{*}\right)}+4 \pi \sum_{a=1}^{g} p_{a} \int^{z_{i}} \omega_{a}\right)\right)
\end{aligned}
$$

where $\mathrm{w}=\left\{w_{k}\right\}, \mathrm{m}=\left\{m_{k}\right\}, \mathrm{p}=\left\{p_{a}\right\}$ and $\mathrm{v}=\left\{\nu_{\alpha}\right\}$ are the filling fractions $\nu_{\alpha} \equiv b g_{s} N_{\alpha}$ which specify the holomorphic integral above. The integrand in (2.19) is a proper onefrom in each variable $z_{i}$ on the covering space of the Riemann surface due to momentum conservation (2.6). The matrix model potential that we find is in the form anticipated by [45].

In order to count the number of moduli of our matrix model we should note that there are $n+2 g-3$ independent filling fractions: naively the number of critical points of the action is $2 g-2+n+1$. However, there are constraints coming from the fact that we are free to move the base point $z^{*}$ and that we have specified the residue at the base point as above by using the momentum conservation. The latter is equivalent to the constraint on the sum of filling fractions $\sum_{\alpha} \nu_{\alpha}=b g_{s} N$. These constraints reduce the number of moduli by two, thus giving the correct counting. The paths of the integrals are defined such that only the solution of (2.18) labeled by the fixed filling fractions $\left\{\nu_{\alpha}\right\}$ contributes to the integrals. The measure factor in (2.19) can be regarded as a generalization of the Vandermonde determinant. The differential $d z \partial_{z} W$ has simple poles with residues $\left(\left\{2 m_{k}\right\},-2 \sum_{k} m_{k}\right)$ at the points $\left(\left\{w_{k}\right\}, z^{*}\right)$.

The integral in (2.13) is then obtained by integrating (2.19) and its complex conjugate over the filling fractions. Thus, in the large $N$ limit, $A_{N}$ becomes

$$
A_{N}=\int_{-\infty}^{\infty} \prod_{a=1}^{g} d p_{a} \int^{n+2 g-3} \prod_{k=1}^{g} d \nu_{k}\left|\exp \left(2 \pi i \sum_{a=1}^{g} \sum_{b=1}^{g} p_{a} \tau_{a b} p_{b}\right) C(\mathrm{w}, \mathrm{m}, \mathrm{p}, \xi) Z_{N}^{\mathcal{C}_{g, n}}(\mathrm{w}, \mathrm{m}, \mathrm{p}, \mathrm{v})\right|^{2}
$$

At the level of the generalized matrix model, the filling fractions $\nu_{\alpha}$ are free parameters. Together with $p_{a}(a=1, \ldots, g)$ which are independent parameters in the potential, we have totally $n+3 g-3$ independent moduli which are identified with the internal momenta in the Liouville conformal block and then with the Coulomb moduli of the gauge theory. The explicit correspondence of these parameters with the internal momenta is discussed in detail in section 4.1. Under this identification, we see from $(2.20)$ that $Z_{N}^{\mathcal{C}_{g, n}}$ (w, m, p, v) is proportional to the conformal block of Liouville theory [71]. 


\section{Degenerations}

To study the behavior of the generalized matrix model when approaching perturbative corners in the space of gauge couplings, we have to study what happens when we degenerate $\mathcal{C}_{g, n}$. The degeneration is usually described by using the plumbing fixture decomposition of the curve. Let $\mathcal{U}_{t}$ be the annulus

$$
\mathcal{U}_{t}=\{(z, w)|z w=t ;| t|<| z|<1 ;| t|<| w \mid<1\}
$$

which as $t \sim 0$ describes the squeezed cylinder. The curve undergoes the decomposition $\mathcal{C}_{g, n}=\mathcal{C}_{g-1, n, 2} \cup \mathcal{U}_{t}$ when the degeneration is of pinching type and $\mathcal{C}_{g, n}=\mathcal{C}_{g_{1}, n_{1}, 1} \cup \mathcal{U}_{t} \cup \mathcal{C}_{g_{2}, n_{2}, 1}$ with $g_{1}+g_{2}=g$ and $n_{1}+n_{2}=n$, with $1-2 g_{i}-n_{i}<0$, when the degeneration is dividing. The components $\mathcal{C}_{g, n, h}$ are here Riemann surfaces with genus $g, n$ punctures and $h$ non overlapping disks removed which will become the punctures in the degeneration limit. The fact that the holomorphic integrals react correctly under the degeneration of the curve $\mathcal{C}_{g, n}$ is a remnant of the analogous property of the conformal field theory [72] and is indeed a consequence of the construction we performed in the previous section. We assume the shrinking cycle do not intersect the contour system along which (2.19) is evaluated.

Let us focus on the dividing case first. In this case the prime form $E\left(z, z^{\prime}\right)$ behaves as follows. If both its arguments belong to a given same component, the prime form reduces to the prime form on that component, while if its arguments belong to different components, then $E\left(z^{\prime}, z^{\prime \prime}\right) \sim E_{1}\left(z^{\prime}, P_{1}\right) E_{2}\left(P_{2}, z^{\prime \prime}\right) t^{-1 / 2}$, where $P_{1,2}$ correspond to the punctures created by the dividing. To see what the prime form degeneration implies for the generalized matrix model measure and potential, we have to split the integration contours in components according to the dividing decomposition. This splits the $\left\{z_{i}\right\}$ in two sets according to which components of the contour they are integrated along, namely $N^{\prime}$ of them on the first component and $N^{\prime \prime}$ on the second with $N=N^{\prime}+N^{\prime \prime}$. Correspondingly, also the puncture set will split in two subsets $w=w^{\prime} \cup$ w", one for each component. By using the above degeneration formulas for the prime form and the fact that the holomorphic harmonic differentials $\omega_{a}$ reduce to the ones relative to the two splitting factors, we get that

$$
Z_{N}^{\mathcal{C}_{g, n}}(\mathrm{w}, \mathrm{m}, \mathrm{p}, \mathrm{v}) \sim Z_{N^{\prime}}^{\mathcal{C}_{g_{1}, n_{1}+1}}\left(\mathrm{w}^{\prime} \cup P_{1}, \mathrm{~m}^{\prime} \cup m_{1}^{*}, \mathrm{p}^{\prime}, \mathrm{v}^{\prime}\right) Z_{N^{\prime \prime}}^{\mathcal{C}_{g_{2}, n_{2}+1}}\left(\mathrm{w}^{\prime \prime} \cup P_{2}, \mathrm{~m}^{\prime \prime} \cup m_{2}^{*}, \mathrm{p}^{\prime \prime}, \mathrm{v}^{\prime \prime}\right)
$$

where $m_{1}^{*}=b g_{s} N^{\prime}-\sum_{k^{\prime}} m_{k^{\prime}}+g_{s} Q\left(g_{1}-1\right)$ and $m_{2}^{*}=b g_{s} N^{\prime \prime}-\sum_{k^{\prime \prime}} m_{k^{\prime \prime}}+g_{s} Q\left(g_{2}-1\right)$ after using momentum conservation. In the computation of (3.1) one needs to count the two extra zeros for the reference holomorphic differential to be placed at the location of the two resulting punctures. The direct computation of the above mass formulas from the integral (2.19) indeed gives, for example, $m_{1}^{*}=-b g_{s} N^{\prime \prime}-g_{2} g_{s} Q+\sum_{k^{\prime \prime}} m_{k^{\prime \prime}}$ where the first term comes from the generalized Vandermonde, the second from the measure term in the square bracket and the third from the punctures. The computation of $m_{2}^{*}$ is identical. Notice that $m_{1}^{*}+m_{2}^{*}=-g_{s} Q$ corresponding to the fact that the two Liouville insertions generated at the punctures are conjugated and therefore the masses of the two flavors at the two punctures $P_{i}$ are equal. The conformal modulus which gets traded to the mass is the total filling fraction between the two integrals. Formula (3.1) is valid for each dividing degeneration such that $g=g_{1}+g_{2}, n=n_{1}+n_{2}$ with $1-2 g_{i}-n_{i}<0$. 
In the pinching case, the prime form restricts to the one of the degenerate surface together with the holomorphic differentials with zero $\alpha_{g}$-cycle. The left over one scales as $2 \pi i \omega_{g}(z) \sim \partial_{z} \log \frac{E\left(z, P_{1}\right)}{E\left(z, P_{2}\right)}$ up to $O(t)$ terms. In this limit, since $\omega$ in the conformal factor has been chosen to be regular, two of its zeroes will be at the two punctures generated at the pinching node and by direct computation one gets

$$
Z_{N}^{\mathcal{C}_{g, n}}(\mathrm{w}, \mathrm{m}, \mathrm{p}, \mathrm{v}) \sim Z_{N}^{\mathcal{C}_{g-1, n+2}}\left(\mathrm{w} \cup\left\{P_{1}, P_{2}\right\}, \mathrm{m} \cup\left\{m_{+}^{*}, m_{-}^{*}\right\}, \hat{\mathrm{p}}, \mathrm{v}\right)
$$

where $\hat{p}_{a}$ are the momenta in the $g-1$ left over handles and

$$
m_{ \pm}^{*}=-\frac{g_{s} Q}{2} \pm i p_{g}
$$

with $p_{g}$ the momentum in the squeezed one. The two contributions to the above mass formulas arise respectively from the term in the square brackets in the measure and the second term in the potential. Once again, the two masses at the generated punctures are Weyl conjugated $m_{+}^{*}+m_{-}^{*}=-g_{s} Q$. In the pinching case the conformal modulus which is traded for the mass is the momentum flowing in the squeezed handle. The formulas above are general and valid at finite $N$.

In the following subsection we will discuss in detail the punctured torus case as an illustration. One could also study for example the punctured genus two case. This case is special since all degenerations reduce to punctured tori. ${ }^{1}$ We discuss some aspects of the Seiberg-Witten geometry on genus two curves in the appendix.

\subsection{Degenerations of punctured tori}

In this subsection, we concentrate on the pinching degeneration of a torus which leads to a sphere with two more punctures. Associated with the torus with $n$ punctures we can consider a class of quiver gauge theories [2,3] whose particular weak coupling descriptions include a gauge theory with circular quiver. Specifying a particular weak coupling description corresponds to choosing a particular marking (a pants decomposition) of the Riemann surface. This gauge theory will reduce in the pinching of the torus to a linear quiver theory with $n-1 \mathrm{SU}(2)$ gauge groups associated with a sphere with $n+2$ punctures. In what follows, we verify that the generalized matrix model correctly reduces to the Penner type matrix model on the sphere [45].

Since on the torus the canonical bundle is trivial, the choice of a base point is not needed. The prime form is $E(z, w)=\frac{\theta_{1}(z-w \mid \tau)}{\theta_{1}^{\prime}(0 \mid \tau)}$ and therefore the generalized matrix model (2.19) reduces to

$$
Z_{N}^{\mathcal{C}_{1, n}} \sim \int \prod_{i=1}^{N} d z_{i} \prod_{i<j} \theta_{1}\left(z_{i}-z_{j}\right)^{-2 b^{2}} e^{\frac{b}{g_{s}} \sum_{i} W\left(z_{i}\right)},
$$

\footnotetext{
${ }^{1}$ The dividing degeneration $\mathcal{C}_{2, n} \rightarrow \mathcal{C}_{1, n^{\prime}+1} \cup \mathcal{C}_{1, n-n^{\prime}+1}$ generates two punctured tori. Indeed, the genus two prime form in such a degeneration reduces to the relevant $\theta$-functions on the two tori since the period matrix at genus two becomes diagonal in the degeneration limit. In the pinching case $\mathcal{C}_{2, n} \rightarrow \mathcal{C}_{1, n+2}$, the genus two $\theta$-function entering the explicit expression of the prime from as $E(z, w)=\frac{\theta\left(\int_{w}^{z} \vec{\omega}, \tau\right)}{\sqrt{\omega_{\square}(z)} \sqrt{\omega_{\square}(w)}}$ contracts to the torus $\theta$-function times a contribution from the off-diagonal term of the period matrix which cancels in the degeneration limit the square-roots of the abelian differentials appearing in the denominator of the prime form.
} 
up to $z_{i}$-independent factors. The potential is

$$
W(z)=\sum_{k=1}^{n} 2 m_{k} \log \theta_{1}\left(z-w_{k}\right)+4 \pi p z,
$$

and

$$
\theta_{1}(z)=2 \sin (\pi z) \prod_{m=1}^{\infty}\left(1-e^{2 \pi i z} q^{m}\right)\left(1-e^{-2 \pi i z} q^{m}\right)\left(1-q^{m}\right),
$$

with $q=e^{2 \pi i \tau}$. The momentum conservation is given by

$$
-\sum_{k=1}^{n} m_{k}+b g_{s} N=0 \text {. }
$$

Also, the identification of the moduli of the torus and the gauge coupling constants of $\mathrm{SU}(2)$ gauge groups $q_{k}$ is as follows [5, 64]:

$$
e^{2 \pi i\left(w_{1}-w_{2}\right)}=q_{1}, \quad e^{2 \pi i\left(w_{2}-w_{3}\right)}=q_{2}, \quad \ldots, \quad e^{2 \pi i\left(w_{n-1}-w_{n}\right)}=q_{n-1}, \quad e^{2 \pi i \tau}=\prod_{k=1}^{n} q_{k},
$$

which, by fixing $w_{n}=0$, leads to

$$
e^{2 \pi i w_{n-1}}=q_{n-1} \equiv t_{n-1}, \quad e^{2 \pi i w_{n-2}}=q_{n-2} q_{n-1} \equiv t_{n-2}, \quad \ldots, \quad e^{2 \pi i w_{1}}=q_{1} \ldots q_{n-1} \equiv t_{1} .
$$

The mass parameters and one of the Coulomb moduli correspond to $m_{k}$ and $p$ respectively.

Let us consider the pinching degeneration of the torus. We take $\operatorname{Im} \tau \rightarrow \infty$ which corresponds in the gauge theory to the decoupling limit of the $n$-th gauge group $q_{n} \rightarrow 0$. To consider the behavior of the generalized matrix model in this limit, we first observe that the prime form reduces as

$$
\begin{aligned}
(d z)^{-1 / 2}(d w)^{-1 / 2} \frac{\theta_{1}(z-w)}{\theta_{1}^{\prime}(0)} & \rightarrow(d z)^{-1 / 2}(d w)^{-1 / 2} \frac{\sin \pi(z-w)}{\pi} \\
& =(d \xi)^{-1 / 2}(d \zeta)^{-1 / 2}(\xi-\zeta),
\end{aligned}
$$

where in the last line we have changed coordinates to $\xi=e^{2 \pi i z}$ and $\zeta=e^{2 \pi i w}$. It is straightforward to see that the Vandermonde determinant of (3.4) reduces to that of the usual $\beta$-deformed matrix model. The potential also reduces to

$$
W(\xi)=\sum_{k=1}^{n} 2 m_{k} \log \left(\xi-t_{k}\right)+2\left(-g_{s} Q / 2-i p\right) \log \xi,
$$

where we have used (3.9) with $t_{n}=1$. Note that the first term corresponding to the momentum at $\xi=0$ comes from the measure factor $\omega(z)^{1+b^{2}}$ of the generalized matrix model (2.19). By the pinching, the punctures at $\xi=0$ and $\infty$ are created. However, the latter disappeared from the potential, which thus reduces exactly to the Penner type matrix model $[45,63]^{2}$

$$
W(z)=\sum_{k=0}^{n-1} 2 m_{k} \log \left(z-t_{k}\right)+2 m_{n} \log (z-1),
$$

\footnotetext{
${ }^{2}$ The convention here is slightly different from the one in $[45,62,63]$. The momenta are related as $2 m=m_{\mathrm{DV}}$. Our convention leads to the momentum conservation (3.13).
} 
with the momentum conservation

$$
-\sum_{k=0}^{n} m_{k}-m_{\infty}+b g_{s} N=g_{s} Q,
$$

where $m_{\infty}$ is the momentum inserted at infinity. The relation between the parameters $t_{k}$ and the gauge couplings [63] (See also [18]) is the same as the one defined in (3.9) with $t_{0}=0$. It follows from (3.11) that $m_{0}=-g_{s} Q / 2-i p$.

Let us then analyze the momentum conservation under this degeneration. On one hand, in the original generalized matrix model, the conservation is described by (3.7). On the other hand, in the Penner type one, the conservation is (3.13). The momentum at infinity is then

$$
m_{\infty}=-\frac{g_{s} Q}{2}+i p .
$$

These values of the momenta $m_{0}$ and $m_{\infty}$ are the ones which were already derived in the generic analysis of the previous section (3.3). Note that there is a slight difference between the momenta $m_{0}$ and $m_{\infty}$, which however disappears in the large $N$ limit.

The original generalized matrix model has $n-1$ independent filling fractions $\nu_{\alpha}$. Recall that the overall $\sum_{\alpha} \nu_{\alpha}=b g_{s} N$ is constrained by the momentum conservation. Thus, by adding $p$, we have $n$ independent parameters which are identified with the vevs of the vector multiplet scalars. The degeneration limit and the above argument mean that $p$ in the potential is the vev of the $n$-th $\mathrm{SU}(2)$ vector multiplet scalar and some combinations of the filling fractions are the vevs of the other $\mathrm{SU}(2)$ scalars. We will give the precise identification in the large $N$ limit in the next section.

\section{Spectral curve of the generalized matrix model}

In this section, we derive the spectral curve of the generalized matrix model (2.19) in the large $N$ limit and show that it coincides with the Seiberg-Witten curve of the corresponding gauge theory.

In the large $N$ limit, the evaluation of (2.19) reduces to the calculation of the critical points. The condition for criticality is given by

$$
d W\left(z_{i}\right)-2 b g_{s} \sum_{j \neq i} d_{z_{i}} \log \left(\frac{E\left(z_{i}, z_{j}\right)}{E\left(z_{i}, z_{*}\right)}\right)=0,
$$

where the potential $W(z)$ is defined in (2.17) and we have used the momentum conservation (2.6). Then, the prepotential in the large $N$ limit, defined as $\exp \left(\mathcal{F} / g_{s}^{2}\right) \equiv Z$, is given by

$$
\frac{1}{g_{s}^{2}} \mathcal{F}=\frac{b}{g_{s}} \sum_{i} W\left(z_{i}\right)-2 b^{2} \sum_{i<j} \log \left(\frac{E\left(z_{i}, z_{j}\right)}{E\left(z_{i}, z^{*}\right)}\right),
$$

where each "eigenvalue" $z_{i}$ satisfies (4.1).

It is natural to assume that the eigenvalues are distributed in line segments around the critical points of $W(z)$, similarly to the usual matrix model. Indeed the second term in (4.2) 
reduces locally to the standard Coulomb gas potential. We denote the line segments as $C_{\alpha}$ where $\alpha=1, \cdots, n+2 g-2$. We assume that $C_{\alpha}$ do not include the base point $z^{*}$ and the punctures $w_{k}$, at which the potential $W(z)$ diverges. We denote by $N_{\alpha}$ the number of eigenvalues on the line segment $C_{\alpha}$, where $N_{\alpha}$ satisfies $\sum_{\alpha=1}^{n+2 g-2} N_{\alpha}=N$.

Let us introduce the eigenvalue density current $\rho(z)$ supported on $\left\{C_{\alpha}\right\}$ and normalized as $\oint_{C_{\alpha}} \rho(z)=b g_{s} N_{\alpha} \equiv \nu_{\alpha}$. Using the variables introduced above, the prepotential and the condition for criticality are written as

$$
\begin{aligned}
& \mathcal{F}=\int_{\sum_{\alpha} C_{\alpha}} \rho(z) W(z)-\int_{\sum_{\alpha} C_{\alpha}} \int_{\sum_{\alpha} C_{\alpha}} \rho(z) \rho\left(z^{\prime}\right) \log \frac{E\left(z, z^{\prime}\right)}{E\left(z, z^{*}\right)}, \\
& d W(z)-2 \int_{\sum_{\alpha} C_{\alpha}} \rho\left(z^{\prime}\right) d_{z} \log \left(\frac{E\left(z, z^{\prime}\right)}{E\left(z, z^{*}\right)}\right)=0,
\end{aligned}
$$

respectively. Here, $z$ in (4.4) is on either of the line segment $C_{\alpha}$ and the integral is defined as the principal integral.

In order to solve the above condition (4.4), we define the following one form, which is the generalization of the resolvent of the usual matrix model

$$
R(z) \equiv \int_{\sum_{\alpha} C_{\alpha}} \rho\left(z^{\prime}\right) d_{z} \log \left(\frac{E\left(z, z^{\prime}\right)}{E\left(z, z^{*}\right)}\right) .
$$

This "resolvent" is defined at generic points $z$ on the Riemann surface contrary to the second term in (4.4). Note that the resolvent as well as $d W(z)$ are single-valued one-forms on the Riemann surface. The resolvent has cuts at the line segments $C_{\alpha}$ and a simple pole at $z^{*}$. Also, the filling fractions are obtained by integrating the resolvent along the cuts as

$$
\nu_{\alpha}=\frac{1}{2 \pi i} \oint_{C_{\alpha}} R(z) \text {. }
$$

On the line segments $C_{\alpha}$, the resolvent behaves as

$$
\begin{aligned}
& R\left(z+i \varepsilon e^{i \varphi(z)}\right)+R\left(z-i \varepsilon e^{i \varphi(z)}\right)=2 \mathrm{P} \int_{\sum_{\alpha} C_{\alpha}} \rho\left(z^{\prime}\right) d_{z} \log \left(\frac{E\left(z, z^{\prime}\right)}{E\left(z, z^{*}\right)}\right)=d W(z), \\
& R\left(z+i \varepsilon e^{i \varphi(z)}\right)-R\left(z-i \varepsilon e^{i \varphi(z)}\right)=\oint_{z} \rho\left(z^{\prime}\right) d_{z} \log \left(\frac{E\left(z, z^{\prime}\right)}{E\left(z, z^{*}\right)}\right)=-2 \pi i \rho(z)
\end{aligned}
$$

where we take the real number $\varepsilon$ infinitely small and we assume that a properly defined function $\varphi(z)$ exists such that $z+i \varepsilon e^{i \varphi(z)}$ or $z-i \varepsilon e^{i \varphi(z)}$ does not go across the cuts $C_{\alpha}$ when $z$ moves along $C_{\alpha}$. The integral in (4.7) is principal integration, which is given as an average of integral along the path above the singularity and that below the singularity. The resolvent should be determined such that (4.7) and (4.8) are satisfied for $z \in C_{\alpha}$. A candidate of the solution for (4.7) is

$$
R_{0}(z)=\frac{1}{2} d W(z) .
$$

However, it does not reproduce the correct structure of singularity expressed in (4.8). We need singular contributions:

$$
R(z)=\frac{1}{2} d W(z)+R(z)_{\operatorname{sing}},
$$


where (4.7) and (4.8) impose

$$
\begin{aligned}
& R\left(z+i \varepsilon e^{i \varphi(z)}\right)_{\operatorname{sing}}+R\left(z-i \varepsilon e^{i \varphi(z)}\right)_{\operatorname{sing}}=0 . \\
& R\left(z+i \varepsilon e^{i \varphi(z)}\right)_{\operatorname{sing}}-R\left(z-i \varepsilon e^{i \varphi(z)}\right)_{\operatorname{sing}}=-2 \pi i \rho(z) .
\end{aligned}
$$

The above discussion is valid for a generic potential $W(z)$. In the following, we use its explicit form (2.17) to determine the resolvent $R(z)$. Then, we find that

$$
\begin{aligned}
R_{\text {sing }}(z) & =\int_{\sum_{\alpha} C_{\alpha}} \rho\left(z^{\prime}\right) d_{z} \log \left(\frac{E\left(z, z^{\prime}\right)}{E\left(z, z^{*}\right)}\right)-\sum_{k=1}^{n} m_{k} d_{z} \log \left(\frac{E\left(z, w_{k}\right)}{E\left(z, z^{*}\right)}\right)-2 \pi \sum_{a=1}^{g} p_{a} \omega_{a}(z) \\
& =\int_{\sum_{\alpha} C_{\alpha}} \rho\left(z^{\prime}\right) d_{z} \log E\left(z, z^{\prime}\right)-\sum_{k=1}^{n} m_{k} d_{z} \log E\left(z, w_{k}\right)-2 \pi \sum_{a=1}^{g} p_{a} \omega_{a}(z)
\end{aligned}
$$

does not depend on the base point $z^{*}$, where we used the momentum conservation (2.6) and ignored the subleading term in the large $N$ expansion. We see that $R(z)_{\text {sing }}$ has cuts in the regions $C_{\alpha}$ and simple poles with residues $m_{k}$ at $z=w_{k}$. Moreover, it is independent on the base point $z^{*}$ as expected.

From (4.11), we see that the sign of $R(z)_{\text {sing }}$ changes across the cuts. Therefore its square only displays singularities at the punctures $z=w_{k}$. From (4.13) we see that these are at most quadratic poles with coefficients $m_{k}{ }^{2}$. The spectral curve of the generalized matrix model thus reads

$$
R_{\text {sing }}(z)^{2}=\sum_{k=1}^{n} m_{k}^{2} \eta\left(z, w_{k}\right)+\zeta(z)
$$

where $\eta\left(z, w_{k}\right)$ are quadratic Strebel differentials, with double pole at $w_{k}$, and $\zeta(z)$ is a quadratic differential which has at most simple poles at $w_{k} . \zeta(z)$ is determined in terms of $n+3 g-3$ parameters; in particular it depends on the $n+2 g-3$ independent filling fractions $\nu_{\alpha}$ and the $g$ internal momenta $p_{a}$. This form of the curve is the same as that of the Seiberg-Witten curve of quiver gauge theory as a cover of the base Riemann surface $[2,3]$. As discussed in [73], the physical information is included in the Prym variety of the SeibergWitten curve, rather than the Jacobian variety. This reduces the number of independent periods of the Seiberg-Witten differential to $n+3 g-3$, which agrees with the number of the filling fractions and $p_{a}$.

In order for the above argument to be a check of AGT correspondence at all genera, we need to show that the spectral curve is indeed the same as the one proposed, in the Virasoro conformal block side, to be the Seiberg-Witten curve: the insertion of the energymomentum tensor in the conformal block

$$
\left\langle T(z) \prod_{k=1}^{n} V_{\alpha_{k}}\left(w_{k}\right)\right\rangle_{\mathcal{C}_{g}} \rightarrow-\frac{x(z)^{2}}{g_{s}^{2}}\left\langle\prod_{k=1}^{n} V_{\alpha_{k}}\left(w_{k}\right)\right\rangle_{\mathcal{C}_{g}}
$$

in the semi-classical limit, which corresponds to the large $N$ limit in the generalized matrix model. It was claimed that $x d z$ coincides with the Seiberg-Witten differential. This was checked in the cases on a sphere and a torus already in [5]. A useful way to capture 
the energy-momentum tensor insertion is to consider the insertion of the degenerate field $\Phi_{1,2}(z) \equiv e^{-\frac{\tilde{\phi}(z)}{b}}$ in the conformal block. By this insertion, the conformal block satisfies the BPZ equation and can be expanded in $g_{s}$ as [26]

$$
\left\langle\Phi_{1,2}(z) \prod_{k=1}^{n} V_{\alpha_{k}}\left(w_{k}\right)\right\rangle_{\mathcal{C}_{g}} \sim \exp \left(\frac{\mathcal{F}_{0}}{g_{s}^{2}}+\frac{1}{b g_{s}} \int^{z} x\left(z^{\prime}\right) d z^{\prime}+\ldots\right)
$$

Thus, the counterpart of the Seiberg-Witten differential in the Virasoro side can be obtained by calculating

$$
x(z)=b g_{s} \frac{\partial}{\partial z} \log \frac{\left\langle\Phi_{1,2}(z) \prod_{k=1}^{n} V_{\alpha_{k}}\left(w_{k}\right)\right\rangle_{\mathcal{C}_{g}}}{\left\langle\prod_{k=1}^{n} V_{\alpha_{k}}\left(w_{k}\right)\right\rangle_{\mathcal{C}_{g}}}
$$

in the semi-classical limit.

In the following, we rewrite (4.17) in terms of the generalized matrix model by using the discussion in section 2. The corresponding calculation in the case of the sphere has been done in [65]. We use the holomorphic half of the integrand in (2.20) to evaluate the conformal block in (4.17). The conformal block with the insertion is also obtained just by changing $n$ to $n+1$ and by regarding that $m_{n+1}=\frac{g_{s}}{2 b}$ and $w_{n+1}=z$. The momentum conservation (2.6) is slightly modified to

$$
\sum_{k=1}^{n} m_{k}+\frac{g_{s}}{2 b}+g_{s} Q(1-g)=b g_{s} N
$$

By collecting the factor dependent on $z$, we obtain

$$
\begin{aligned}
x(z) & =b g_{s} \frac{\partial}{\partial z} \log \left(\omega(z)^{\frac{1}{4 b^{2}}} \prod_{I} E\left(z, \xi_{I}\right)^{\frac{Q}{2 b}} E\left(z, z^{*}\right)^{N-\frac{\sum_{k} m_{k}}{b g_{s}}} e^{-\frac{W(z)}{2 b g_{s}}}\left\langle\prod_{i} \frac{E\left(z, z_{i}\right)}{E\left(z, z^{*}\right)}\right\rangle\right) \\
& =-\frac{1}{2} \frac{\partial W(z)}{\partial z}+b g_{s} \frac{\partial}{\partial z} \log \left\langle\prod_{i} \frac{E\left(z, z_{i}\right)}{E\left(z, z^{*}\right)}\right\rangle+\mathcal{O}\left(N^{-1}\right),
\end{aligned}
$$

where we have used the deformed momentum conservation (4.18) in the second equality. The expectation value is defined by

$$
\langle\ldots\rangle=\frac{\int \prod d z_{i} \mu e^{\frac{b}{g_{s}} \sum_{i} W\left(z_{i}\right)} \ldots}{\int \prod d z_{i} \mu e^{\frac{b}{g_{s}} \sum_{i} W\left(z_{i}\right)}} .
$$

A subtlety is that the numerator in (4.20) is defined with the deformed momentum conservation (4.18) while the denominator with the original momentum conservation. However, note that the effect of the insertion of the degenerate field is a subleading contribution in the large $N$ limit, as in (4.16). Note also that the deformation of the momentum conservation gives rise to that of the prepotential $\mathcal{F}_{0}$, which does not depend on $z$ and disappears by the partial derivative in terms of $z$. Thus, we can use the same external momenta $m_{k}$ and the same $N$ as those of the denominator of (4.20) to evaluate the numerator in the expectation value. Also, some of the internal momenta in the numerator are shifted by $\pm b / 2$ depending on where we insert the degenerate operator as discussed in [26], but again, 
the effect of this shift does not produce the factor dependent on $z$ in the large $N$ limit. Thus, the expectation value in (4.19) can be evaluated by substituting the solution of the condition of criticality (4.1) or (4.4), which leads to

$$
b g_{s} \frac{\partial}{\partial z} \log \left\langle\prod_{i} \frac{E\left(z, z_{i}\right)}{E\left(z, z^{*}\right)}\right\rangle=b g_{s}\left\langle\sum_{i} \frac{\partial}{\partial z} \log \frac{E\left(z, z_{i}\right)}{E\left(z, z^{*}\right)}\right\rangle+\mathcal{O}\left(N^{-1}\right) .
$$

From the discussion above, we finally obtain

$$
x(z) d z \sim-\frac{d W(z)}{2}+R(z)=R_{\operatorname{sing}}(z),
$$

up to $\mathcal{O}\left(N^{-1}\right)$ terms. This shows that the resolvent $R_{\text {sing }}$ is indeed $x(z) d z$ in (4.15), which was claimed to agree with the Seiberg-Witten differential.

\subsection{Dependence on internal momenta}

In this subsection, we show that the spectral curve of the generalized matrix model depends on the Coulomb moduli parameters in the same way as the Seiberg-Witten curve, which completes the check of AGT correspondence at all genera. In order to do that, we first have to specify the marking of the Riemann surface $\mathcal{C}_{g, n}$, which is done by choosing $n+3 g-3$ physically independent cycles. This marking determines the conformal block labeled by a trivalent graph on one hand, and also the corresponding weak coupling description of the gauge theory on the other hand. On the gauge theory side, each vacuum expectation value of each vector multiplet scalar is obtained by an integral along each cycle of the marking $\gamma_{r}$ :

$$
a_{r}=\frac{1}{2 \pi i} \oint_{\gamma_{r}} \lambda_{\mathrm{SW}}
$$

where $\lambda_{\mathrm{SW}}$ is the Seiberg-Witten differential. We would like to show that the spectral curve also satisfies the corresponding relation.

Before going to that issue, we show in the following discussion that each pair of pants has one cut under the marking. In general, three-punctured sphere always has one cut [47] because the classical potential of the corresponding Penner type matrix model with generic mass parameters has one extremum. We can also show this by considering the dividing limit of four-punctured sphere: under the decomposition, it is known that one cut exists in the pants including the punctures originally at 0 and $q$, while the other cut exist in the pants including the punctures at 1 and $\infty$. Furthermore, unless the mass parameters vanish, there are no punctures belonging to the cut. This indicates that three-punctured sphere with generic mass parameters has one cut apart from the three punctures. Since we have shown that our generalized matrix model behaves correctly in the degeneration limit, the cuts of its resolvent should be placed in such a way that each pair of pants has one cut.

Once the marking is specified, an explicit correspondence should be determined between the $n+3 g-3$ internal momenta of the Virasoro conformal block and the $n+2 g-3$ independent filling fractions together with the $n$ parameters $p_{a}$ in the potential. In order to see this explicitly, we can use the results on the degeneration obtained in section 3. Note that among the intermediate states in the conformal block, only the primary state remains 
and appears as the external state in the degeneration limit. Thus, the external momentum appeared by the degeneration which shrinks a particular cycle can be seen as the internal momentum of the corresponding place before the degeneration. In order to determine all the internal momenta, we pinch all the $A$-cycles of the Riemann surface $\mathcal{C}_{g, n}$ until it becomes $\mathcal{C}_{0, n+2 g}$ and then divide $\mathcal{C}_{0, n+2 g}$ until they split into three-punctured spheres in a way that it reproduces the specified pants decomposition. Whichever marking we take, we can read off all the internal momenta by considering the corresponding degeneration limit.

The $A$-cycles of $\mathcal{C}_{g, n}$ can be chosen to coincide with some of $\gamma_{r}$ 's. Thus, the set $\left\{\gamma_{r}\right\}$ can be divided as $\left\{\gamma_{r}\right\}=\left\{A_{a}\right\} \cup\left\{\gamma_{\alpha}\right\}$ where $a=1, \ldots, g$ and $\alpha=1, \ldots, n+2 g-3$, such that $A$-cycles of $\mathcal{C}_{g, n}$ are $A_{a}$ and $\gamma_{\alpha}$ are the remaining cycles. By shrinking $A_{a}$-cycles, we obtain (3.3). Especially, in the large $N$ limit, the internal momenta (multiplied by $g_{s}$ ) corresponding to the $A_{a}$ cycles of the Riemann surface $\mathcal{C}_{g}$ are given by

$$
a_{a}=i p_{a} . \quad(a=1, \cdots, g)
$$

After pinching all the $A_{a}$ cycles, the remaining internal momenta can be obtained as written just below (3.1) by further considering the dividing limit which shrinks $\gamma_{\alpha}$ cycles. Since each pair of pants has one cut, on which $N_{\beta}$ eigenvalues $\left\{z_{i}\right\}$ exist, the number of eigenvalues $N^{\prime}$ or $N^{\prime \prime}$ on each side of the dividing component of the Riemann surface can be written in terms of sum of the filling fractions. In the large $N$ limit, the internal momenta (multiplied by $g_{s}$ ) flowing through $\gamma_{\alpha}$ cycles are given by

$$
a_{\alpha}=\sum_{\beta} \nu_{\beta}-\sum_{k} m_{k}+\sum_{a}\left( \pm i p_{a}\right), \quad(\alpha=1, \cdots, n+2 g-3)
$$

where the range of the sum depends which $\gamma_{\alpha}$ cycle we shrink under the marking of the Riemann surface. The third term is the contribution from new punctures which appear by pinching $A_{a}$ cycles in the previous step. The sign \pm is determined for each puncture according to the direction of the $A_{a}$ cycle. Although the internal momenta (4.24) and (4.25) are obtained in the degeneration limit, we assume that these relations hold for arbitrary moduli parameters of the Riemann surface $\mathcal{C}_{g, n}$ because the filling fraction $\nu_{\alpha}$ and the parameter $p_{a}$ are independent of the moduli parameters. It is remarkable that the form of our generalized matrix model in the large $N$ limit is universal for any choice of markings of the Riemann surface. However, the identification of the parameters with internal momenta depend on such choice, which reflects in the difference of the conformal blocks labeled by different trivalent graphs.

So far, we have discussed the relation between the internal momenta of the Virasoro conformal block and the independent filling fractions and parameters $p_{a}$. In the following, we check that the corresponding relation as (4.23) is reproduced from the spectral curve under these identification of the parameters. By using (4.13), we can explicitly calculate the $A_{a}$-cycle integrals as

$$
\frac{1}{2 \pi i} \int_{A_{a}} R_{\text {sing }}=i p_{a} \quad(a=1, \cdots, g)
$$




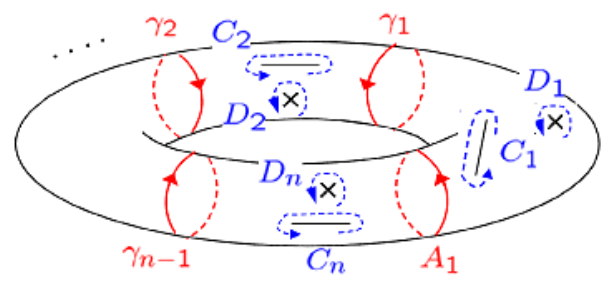

Figure 1. A marking of $\mathcal{C}_{1, n}$ corresponding to the elliptic quiver and the $A_{1}$ - and $\gamma_{\alpha}$-cycles. E.g., $\gamma_{1}=A_{1}+C_{1}+D_{1}$ and $\gamma_{2}=A_{1}+C_{1}+C_{2}+D_{1}+D_{2}$.

Note that the convention here is $\oint_{A_{a}} \omega_{b}=\delta_{b}^{a}$. Taking into account the identification of the parameters (4.24), we see that the corresponding relation as (4.23) for $A_{a}$-cycle is confirmed.

For the $\gamma_{\alpha}$ cycles, the check is not direct, because $\gamma_{\alpha}$ are not the contours $C_{\alpha}$ along the cuts of the spectral curve of the matrix model. Recall that there are indeed $n+2 g-2$ cuts in the spectral curve, but the integral of the resolvent along one of them is not independent due to the momentum conservation. We will call the remaining $n+2 g-3$ cuts the independent ones. It follows that the cycles around the independent cuts, which we have denoted by $C_{\alpha}$ $(\alpha=1, \cdots n+2 g-3)$, can be expressed as sum of the three cycles which go around each leg of the pants. That is, $C_{\alpha}$ can be written as a linear combination of $\gamma_{r}(r=1, \cdots n+3 g-3)$ and $D_{k}(k=1, \cdots n)$, where $D_{k}$ are the contours around the simple poles of $R_{\text {sing. Thus, }} \gamma_{\alpha}$ $(\alpha=1, \cdots n+2 g-3)$ can be written as $\gamma_{\alpha}=n_{\alpha}{ }^{a} A_{a}+c_{\alpha}{ }^{\beta} C_{\beta}+s_{\alpha}{ }^{k} D_{k}$, where the coefficients $n_{\alpha}{ }^{a}, c_{\alpha}{ }^{\beta}$ and $s_{\alpha}{ }^{k}$ are integers. (Note that in addition to them there could exist a cycle around the base point. However, when we consider $R_{\text {sing }}$, this dependence disappears in the large $N$ limit, as stated in (4.13).) In terms of these, we calculate the integral over $\gamma_{\alpha}$ cycles as

$$
\begin{aligned}
\frac{1}{2 \pi i} \oint_{\gamma_{\alpha}} R_{\operatorname{sing}}(z) & =\frac{1}{2 \pi i} \oint_{\gamma_{\alpha}}\left[R(z)-\sum_{k=1}^{n} m_{k} \omega_{w_{k}, z^{*}}(z)-2 \pi \sum_{a=1}^{g} p_{a} \omega_{a}(z)\right] \\
& =\sum_{\beta} c_{\alpha}{ }^{\beta} \nu_{\beta}-\sum_{k} s_{\alpha}{ }^{k} m_{k}+i \sum_{a} n_{\alpha}{ }^{a} p_{a}
\end{aligned}
$$

where $\omega_{x, y}(z)=d_{z} \log \frac{E(z, x)}{E(z, y)}$ is the Abelian differential of the third kind.

To determine the coefficients, we briefly show the example below. In the case of the torus $\mathcal{C}_{1, n}$, there is only one $A$-cycle and the number of independent cuts is $n-1$. For $n=1$, there is no independent cut and the parameter $p$ is identified with the vacuum expectation value of the vector multiplet scalar of $\mathcal{N}=2^{*}$ gauge theory as analyzed in [64]. For $n>1$, we consider the marking corresponding to $\mathrm{SU}(2)^{n}$ elliptic quiver gauge theory and choose the $A_{1}$-cycle and $\gamma_{\alpha}$-cycles as depicted in figure 1 . As stated above, each pair of pants has one cut. (This can also be explicitly checked by solving the equations of motion in the weak coupling limit.) Therefore, it is easy to obtain the coefficients above $n_{\alpha}^{1}=1$ and

$$
c_{\alpha}^{\beta}=\left\{\begin{array}{l}
1(\beta=1, \ldots, \alpha) \\
0(\beta=\alpha+1, \ldots, n-1)
\end{array} \quad s_{\alpha}^{k}=\left\{\begin{array}{l}
1(k=1, \ldots, \alpha) \\
0(k=\alpha+1, \ldots, n-1)
\end{array}\right.\right.
$$


This indicates

$$
\frac{1}{2 \pi i} \oint_{\gamma_{\alpha}} R_{\operatorname{sing}}(z)=\sum_{\beta=1}^{\alpha} \nu_{\beta}-\sum_{k=1}^{\alpha} m_{k}+i p
$$

in addition to the one obtained by the $A$-cycle integral (4.26). Comparing it with the result obtained from (4.25), we see that it exactly reproduces the internal momenta flowing through $\gamma_{\alpha}$ cycle. Similarly, it is straightforward to check for generic case that (4.27) coincides with that obtained from (4.25). Thus, we have confirmed that the corresponding relations as (4.23) for $\gamma_{\alpha}$ cycles are reproduced.

\section{Conclusions}

In this paper, we have shown that the perturbative analysis of Liouville correlation functions displays in the large $N$ limit holomorphic factorization of the surface integrals and leads to generalized matrix models, defined on the cover of $\mathcal{C}_{g, n}$, which describe the relevant Virasoro conformal blocks. We provided an all genera check of the AGT correspondence by obtaining the Seiberg-Witten data from the saddle point analysis of these generalized matrix models.

We underline that the models presented in this paper could be useful for the exploration of the full set of gauge theories with generalized quiver structure of [3]. Indeed, so far most of the analysis of the AGT correspondence has been focused on the linear and elliptic quiver cases, mainly due to the lack of calculational tools for higher genera. However, to fully exploit the generalized matrix model approach one should be able to extend its analysis to finite $N$. This would amount to provide a full derivation at finite $N$ of the holomorphic factorization, which in turn would give a precise prescription for the contour integrals possibly extending the recipe of $[48-50,66]$ to higher genera.

Notice anyway that our large $N$ analysis depends only on the homotopy class of the contours via the filling fractions as explained in section 4.1. In this sense our results are universal with respect to a particular choice of contour's representatives in the matrix integral.

Moreover, for gauge groups of higher rank, which according to [45, 46] should correspond to multi-matrix models, this approach could shed light on the description of strongly coupled sectors naturally appearing in the general framework and not admitting a known lagrangian description.

Another very interesting issue to explore is the relation of the generalized matrix models with the quantization of integrable systems [42, 74-80]. (See also [81-85].) In particular this could provide an alternative derivation of the quantum Hamiltonians for Hitchin integrable systems and generalize it to higher genera.

\section{Acknowledgments}

A.T. thanks R. Santachiara for useful discussions. F.Y. thanks T. Eguchi and N. Nekrasov for useful discussions. G.B. is partially supported by the INFN project TV12. The research of K.M. is supported in part by JSPS Bilateral Joint Projects (JSPS-RFBR collaboration). A.T. is partially supported by PRIN "Geometria delle varietà algebriche e loro spazi di moduli" and the INFN project PI14 "Nonperturbative dynamics of gauge theories". 


\section{A Addendum: playing with genus 2 curves}

A hyperelliptic curve $\mathcal{C}_{g}$ of genus $g$ is given by the equation

$$
y^{2}=P_{2 g+2}(x)
$$

where $P_{2 g+2}$ is a polynomial of degree $2 g+2$ and is realized ${ }^{3}$ in the total space of $T \mathbb{P}^{\frac{g+1}{2}}$.

As it is well known, all genus 2 curves are hyperelliptic. These are realized in general by a sextic polynomial equation

$$
y^{2}=\prod_{i=1}^{6}\left(x-a_{i}\right)
$$

which we denote by $\mathcal{C}_{2}$.

The complex structure moduli $\overline{\mathcal{M}}_{2,0}$ of genus two curves is then obtained by considering the complex parameters $\left\{a_{i}\right\}$ modulo the action of the permutation group $S_{6}$ and the $\operatorname{PSL}(2, \mathbf{C})$.

A basis of abelian differentials is given by $\omega_{a}=\frac{x^{a-1} d x}{y}, a=1,2$, while a basis of quadratic differentials is given by $\phi_{\alpha}=\frac{x^{\alpha-1} d x^{2}}{y^{2}}, \alpha=1,2,3$ [86].

The Seiberg-Witten (SW) geometry of the $\mathrm{SU}(2)$ theory at genus 2 is specified by a double cover of $\mathcal{C}_{2}$ in $T^{*} \mathcal{C}_{2}$. As such, this is specified by a general quadratic differential on $\mathcal{C}_{2}$ in the form

$$
w^{2}=\Phi_{2}
$$

where $\Phi_{2}=\sum_{\alpha} K_{\alpha} \phi_{\alpha}$ can be expanded in the Coulomb moduli $K_{\alpha}$ of the theory.

The perturbative expansions of the theory are available in the vicinity of the degeneration locus of the moduli space, namely around

$$
\partial \overline{\mathcal{M}}_{2,0}=\overline{\mathcal{M}}_{1,1} \times \overline{\mathcal{M}}_{1,1} \cup \overline{\mathcal{M}}_{1,2}
$$

The second factor in (A.3) is still generically not lagrangian and has to be degenerated as $\partial \overline{\mathcal{M}}_{1,2}=\overline{\mathcal{M}}_{1,1} \times \overline{\mathcal{M}}_{0,3} \cup \overline{\mathcal{M}}_{0,4}$ to reach corners around which known lagrangian descriptions are available. The first factor in (A.3), being given by two copies of the $\mathcal{N}=2^{*} \mathrm{SU}(2)$ theory, is already lagrangian. The first degeneration is dividing and the second one is pinching.

Let us discuss the dividing case in detail. This is reached by taking the limit in which three branch points in (A.1) collide. To be concrete, let's fix the position of two of them at 0 and $\infty$, write our curve as

$$
y^{2}=x\left(x-a_{1} \epsilon\right)\left(x-a_{2} \epsilon\right)\left(x-a_{3}\right)\left(x-a_{4}\right)
$$

and take the limit as $\epsilon \rightarrow 0$. The curve (A.4) becomes in the $x$ coordinate

$$
y^{2}=x^{3}\left(x-a_{3}\right)\left(x-a_{4}\right)
$$

\footnotetext{
${ }^{3}$ Under conformal inversion $x=1 / x^{\prime}$ on the Riemann sphere, the stability of the description is guaranteed by the transformation $y=y^{\prime} x^{\prime-(g+1)}=y^{\prime}\left(\frac{\partial x}{\partial x^{\prime}}\right)^{\frac{g+1}{2}}$.
} 
which, redefining $y=x \tilde{y}$, reads

$$
\tilde{y}^{2}=x\left(x-a_{3}\right)\left(x-a_{4}\right)
$$

that is the torus with a puncture at $x=0$.

Let us check now the SW geometry in the degeneration limit. The issue to discuss is just the scaling of the Coulomb parameters in this limit. Notice that the degeneration is obtained by contracting to zero two nearby branching points, therefore we are saturating a complex structure modulus corresponding to a Beltrami differential $\mu_{\epsilon}$ with support around the origin of size $\sim \epsilon$. This is dual to a holomorphic quadratic differential which, not to have a vanishing overlap integral with $\mu_{\epsilon}$ should not be zero at $x=0$. This is uniquely determined to be $\frac{d x^{2}}{y^{2}}$. Therefore, along the limit with $\epsilon \rightarrow 0$, the corresponding parameter in the SW curve has to scale away.

As a consequence of the above reasoning, exposing the $\epsilon$-parameter, the SW curve is parametrized as

$$
w^{2}=\frac{u^{\prime} \epsilon+m^{2} x+u x^{2}}{y^{2}}(d x)^{2}
$$

The degeneration of $\mathcal{C}_{2}$ is easily kept into account in the SW geometry which becomes

$$
w^{2}=\frac{m^{2} x^{-1}+u}{\tilde{y}^{2}}(d x)^{2}
$$

The standard parameterization of the punctured torus is in the coordinates where the puncture sits at $\infty$. Therefore, we rewrite the elliptic curve (A.5) after the inversion $x=\frac{1}{x^{\prime}}$ to pull the puncture at $x^{\prime}=\infty$ and redefine accordingly $\tilde{y}=\frac{1}{\left(x^{\prime}\right)^{2}} \tilde{y}^{\prime}$. After this, the SW curve reads

$$
w^{2}=\frac{m^{2} x^{\prime}+u}{\left(\tilde{y}^{\prime}\right)^{2}}\left(d x^{\prime}\right)^{2}
$$

which we can put in the representation with respect to the periodic coordinate via the Weierstrass parameterization ${ }^{4} x^{\prime}=\mathcal{P}(z)+c$ and $\tilde{y}^{\prime}=\frac{d}{d z} \mathcal{P}(z)$ so that we stay with

$$
w^{2}=\left[m^{2} \mathcal{P}(z)+\left(u+c m^{2}\right)\right](d z)^{2}
$$

which is the SW curve for a copy of the $\mathcal{N}=2^{*}$ theory.

The other copy corresponds to the other half in which the original genus 2 surface was split. Let's see how to get this second copy. In order to do it we have to consider the curve in the coordinate appropriate for the other half, namely we have to change (A.4) to $x=\epsilon / \hat{x}$ and correspondingly $y=\hat{y} \frac{\epsilon^{3 / 2}}{(\hat{x})^{3}}$ after which we get

$$
\hat{y}^{2}=\hat{x}\left(1-a_{1} \hat{x}\right)\left(1-a_{2} \hat{x}\right)\left(\epsilon-a_{3} \hat{x}\right)\left(\epsilon-a_{4} \hat{x}\right) .
$$

(A.9) becomes after the degeneration limit the curve

$$
\hat{y}^{2}=\hat{x}^{3}\left(1-a_{1} \hat{x}\right)\left(1-a_{2} \hat{x}\right)\left(a_{3} a_{4}\right)
$$

\footnotetext{
${ }^{4}$ The constant $c$ needs to bring (A.5) to the standard Weierstrass form where the quadratic term vanishes and can be computed explicitly.
} 
which we bring to the form of a punctured torus by redefining $\hat{y}=\tilde{\hat{y}} \hat{x}$ and get

$$
\tilde{\hat{y}}^{2}=\hat{x}\left(1-a_{1} \hat{x}\right)\left(1-a_{2} \hat{x}\right)\left(a_{3} a_{4}\right) .
$$

Let's follow now what happens to the SW curve (A.6) in the $\hat{x}$ patch. This becomes

$$
w^{2}=\frac{u^{\prime}+m^{2} \hat{x}^{-1}+u \epsilon \hat{x}^{-2}}{\hat{y}^{2}}(d \hat{x})^{2}
$$

which in the limit $\epsilon \rightarrow 0$ has the same form of (A.7), but referring to the second punctured torus with an independent Coulomb parameter $u^{\prime}$. So, following the same procedure leading to (A.8), we get the second copy of $\mathcal{N}=2^{*}$ with an independent gauge coupling and Coulomb parameter but the same mass as the first.

A pinching of the genus 2 curves (A.4) can be obtained by letting $\epsilon \rightarrow \frac{a_{3}}{a_{2}}$ for example. In such a case the curve gets to

$$
y^{2}=x\left(x-a_{1} a_{3} / a_{2}\right)\left(x-a_{3}\right)^{2}\left(x-a_{4}\right)
$$

which is, after renaming $y=\tilde{y}\left(x-a_{3}\right)$, the twice punctured torus

$$
\tilde{y}^{2}=x\left(x-a_{1} a_{3} / a_{2}\right)\left(x-a_{4}\right) .
$$

Correspondingly, the holomorphic quadratic differential entering the Seiberg-Witten curve (A.6) becomes

$$
\Phi_{2} \rightarrow \frac{u^{\prime} a_{3} / a_{2}+m^{2} x+u x^{2}}{\left(x-a_{3}\right)^{2} \tilde{y}^{2}}(d x)^{2}
$$

which explicitly displays quadratic poles at the two images of $x=a_{3}$ with equal coefficients. This coefficient is actually fixed by the Coulomb parameter corresponding to the ungauged group $\mathrm{SU}(2)$ at the end of the shrinking of the handle.

Open Access. This article is distributed under the terms of the Creative Commons Attribution Noncommercial License which permits any noncommercial use, distribution, and reproduction in any medium, provided the original author(s) and source are credited.

\section{References}

[1] A. Klemm, W. Lerche, P. Mayr, C. Vafa and N.P. Warner, Self-dual strings and $N=2$ supersymmetric field theory, Nucl. Phys. B 477 (1996) 746 [hep-th/9604034] [SPIRES].

[2] E. Witten, Solutions of four-dimensional field theories via M-theory, Nucl. Phys. B 500 (1997) 3 [hep-th/9703166] [SPIRES].

[3] D. Gaiotto, $N=2$ dualities, arXiv:0904.2715 [SPIRES].

[4] N.A. Nekrasov, Seiberg-Witten prepotential from instanton counting, Adv. Theor. Math. Phys. 7 (2004) 831 [hep-th/0206161] [SPIRES].

[5] L.F. Alday, D. Gaiotto and Y. Tachikawa, Liouville correlation functions from four-dimensional gauge theories, Lett. Math. Phys. 91 (2010) 167 [arXiv:0906.3219] [SPIRES]. 
[6] D. Gaiotto, Asymptotically free $N=2$ theories and irregular conformal blocks, arXiv:0908.0307 [SPIRES].

[7] A. Marshakov, A. Mironov and A. Morozov, On non-conformal limit of the AGT relations, Phys. Lett. B 682 (2009) 125 [arXiv:0909.2052] [SPIRES].

[8] A. Marshakov, A. Mironov and A. Morozov, Zamolodchikov asymptotic formula and instanton expansion in $N=2$ SUSY $N_{f}=2 N_{c}$ QCD, JHEP 11 (2009) 048 [arXiv: 0909.3338] [SPIRES].

[9] R. Poghossian, Recursion relations in CFT and $N=2$ SYM theory, JHEP 12 (2009) 038 [arXiv:0909.3412] [SPIRES].

[10] A. Mironov and A. Morozov, Proving AGT relations in the large-c limit, Phys. Lett. B 682 (2009) 118 [arXiv:0909.3531] [SPIRES].

[11] V. Alba and A. Morozov, Non-conformal limit of AGT relation from the 1-point torus conformal block, JETP Lett. 90 (2009) 708 [arXiv:0911.0363] [SPIRES].

[12] L. Hadasz, Z. Jaskolski and P. Suchanek, Recursive representation of the torus 1-point conformal block, JHEP 01 (2010) 063 [arXiv:0911.2353] [SPIRES].

[13] V.A. Fateev and A.V. Litvinov, On AGT conjecture, JHEP 02 (2010) 014 [arXiv: 0912.0504] [SPIRES].

[14] G. Giribet, On triality in $N=2$ SCFT with $N_{f}=4$, JHEP 01 (2010) 097 [arXiv:0912.1930] [SPIRES].

[15] R. Santachiara and A. Tanzini, Moore-Read fractional quantum Hall wavefunctions and SU(2) quiver gauge theories, Phys. Rev. D 82 (2010) 126006 [arXiv:1002.5017] [SPIRES].

[16] L. Hadasz, Z. Jaskolski and P. Suchanek, Proving the AGT relation for $N_{f}=0,1,2$ antifundamentals, JHEP 06 (2010) 046 [arXiv: 1004.1841] [SPIRES].

[17] D. Nanopoulos and D. Xie, $N=2$ generalized superconformal quiver gauge theory, arXiv: 1006.3486 [SPIRES].

[18] V. Alba and A. Morozov, Check of AGT relation for conformal blocks on sphere, Nucl. Phys. B 840 (2010) 441 [arXiv:0912.2535] [SPIRES].

[19] N. Wyllard, $A_{N-1}$ conformal Toda field theory correlation functions from conformal $N=2$ $\mathrm{SU}(N)$ quiver gauge theories, JHEP 11 (2009) 002 [arXiv:0907.2189] [SPIRES].

[20] A. Mironov and A. Morozov, On AGT relation in the case of $\mathrm{U}(3)$, Nucl. Phys. B 825 (2010) 1 [arXiv:0908.2569] [SPIRES].

[21] D. Nanopoulos and D. Xie, Hitchin equation, singularity and $N=2$ superconformal field theories, JHEP 03 (2010) 043 [arXiv: 0911.1990] [SPIRES].

[22] S. Kanno, Y. Matsuo, S. Shiba and Y. Tachikawa, $N=2$ gauge theories and degenerate fields of Toda theory, Phys. Rev. D 81 (2010) 046004 [arXiv:0911.4787] [SPIRES].

[23] M. Taki, On AGT conjecture for pure super Yang-Mills and W-algebra, JHEP 05 (2011) 038 [arXiv: 0912.4789] [SPIRES].

[24] D. Nanopoulos and D. Xie, Hitchin equation, irregular singularity and $N=2$ asymptotical free theories, arXiv:1005.1350 [SPIRES].

[25] S. Kanno, Y. Matsuo and S. Shiba, Analysis of correlation functions in Toda theory and AGT-W relation for $\mathrm{SU}(3)$ quiver, Phys. Rev. D 82 (2010) 066009 [arXiv:1007.0601] [SPIRES]. 
[26] L.F. Alday, D. Gaiotto, S. Gukov, Y. Tachikawa and H. Verlinde, Loop and surface operators in $N=2$ gauge theory and Liouville modular geometry, JHEP 01 (2010) 113 [arXiv:0909.0945] [SPIRES].

[27] N. Drukker, J. Gomis, T. Okuda and J. Teschner, Gauge theory loop operators and Liouville theory, JHEP 02 (2010) 057 [arXiv: 0909.1105] [SPIRES].

[28] A. Gadde, E. Pomoni, L. Rastelli and S.S. Razamat, S-duality and 2d topological QFT, JHEP 03 (2010) 032 [arXiv:0910.2225] [SPIRES].

[29] J.-F. Wu and Y. Zhou, From Liouville to Chern-Simons, alternative realization of Wilson loop operators in AGT duality, arXiv:0911.1922 [SPIRES].

[30] N. Drukker, D. Gaiotto and J. Gomis, The virtue of defects in $4 D$ gauge theories and $2 D$ CFTs, JHEP 06 (2011) 025 [arXiv: 1003.1112] [SPIRES].

[31] F. Passerini, Gauge theory Wilson loops and conformal Toda field theory, JHEP 03 (2010) 125 [arXiv: 1003.1151] [SPIRES].

[32] C. Kozcaz, S. Pasquetti and N. Wyllard, A $\& 3$ B model approaches to surface operators and Toda theories, JHEP 08 (2010) 042 [arXiv: 1004.2025] [SPIRES].

[33] L.F. Alday and Y. Tachikawa, Affine SL(2) conformal blocks from $4 d$ gauge theories, Lett. Math. Phys. 94 (2010) 87 [arXiv: 1005.4469] [SPIRES].

[34] T. Dimofte, S. Gukov and L. Hollands, Vortex counting and Lagrangian 3-manifolds, arXiv: 1006.0977 [SPIRES].

[35] M. Taki, Surface operator, bubbling Calabi-Yau and AGT relation, arXiv:1007.2524 [SPIRES].

[36] H. Awata, H. Fuji, H. Kanno, M. Manabe and Y. Yamada, Localization with a surface operator, irregular conformal blocks and open topological string, arXiv:1008.0574 [SPIRES].

[37] C. Kozcaz, S. Pasquetti, F. Passerini and N. Wyllard, Affine sl $(N)$ conformal blocks from $N=2 \mathrm{SU}(N)$ gauge theories, JHEP 01 (2011) 045 [arXiv: 1008.1412] [SPIRES].

[38] J. Gomis and B. Le Floch, 't Hooft operators in gauge theory from Toda CFT, arXiv: 1008.4139 [SPIRES].

[39] K. Hosomichi, S. Lee and J. Park, AGT on the S-duality wall, JHEP 12 (2010) 079 [arXiv: 1009.0340] [SPIRES].

[40] N. Wyllard, $W$-algebras and surface operators in $N=2$ gauge theories, J. Phys. A 44 (2011) 155401 [arXiv:1011.0289] [SPIRES].

[41] Y. Yamada, A quantum isomonodromy equation and its application to $N=2 \mathrm{SU}(N)$ gauge theories, J. Phys. A 44 (2011) 055403 [arXiv: 1011.0292] [SPIRES].

[42] K. Maruyoshi and M. Taki, Deformed prepotential, quantum integrable system and Liouville field theory, Nucl. Phys. B 841 (2010) 388 [arXiv: 1006.4505] [SPIRES].

[43] G. Bonelli and A. Tanzini, Hitchin systems, $N=2$ gauge theories and $W$-gravity, Phys. Lett. B 691 (2010) 111 [arXiv:0909.4031] [SPIRES].

[44] L.F. Alday, F. Benini and Y. Tachikawa, Liouville/Toda central charges from M5-branes, Phys. Rev. Lett. 105 (2010) 141601 [arXiv:0909.4776] [SPIRES].

[45] R. Dijkgraaf and C. Vafa, Toda theories, matrix models, topological strings and $N=2$ gauge systems, arXiv:0909.2453 [SPIRES]. 
[46] H. Itoyama, K. Maruyoshi and T. Oota, The quiver matrix model and 2d-4d conformal connection, Prog. Theor. Phys. 123 (2010) 957 [arXiv:0911.4244] [SPIRES].

[47] R. Schiappa and N. Wyllard, An Ar threesome: matrix models, 2d CFTs and $4 d \mathrm{~d}=2$ gauge theories, arXiv:0911.5337 [SPIRES].

[48] A. Mironov, A. Morozov and S. Shakirov, Matrix model conjecture for exact BS periods and Nekrasov functions, JHEP 02 (2010) 030 [arXiv:0911.5721] [SPIRES].

[49] A. Mironov, A. Morozov and S. Shakirov, Conformal blocks as Dotsenko-Fateev integral discriminants, Int. J. Mod. Phys. A 25 (2010) 3173 [arXiv:1001.0563] [SPIRES].

[50] H. Itoyama and T. Oota, Method of generating q-expansion coefficients for conformal block and $N=2$ Nekrasov function by beta-deformed matrix model, Nucl. Phys. B 838 (2010) 298 [arXiv:1003.2929] [SPIRES].

[51] M. Fujita, Y. Hatsuda and T.-S. Tai, Genus-one correction to asymptotically free Seiberg-Witten prepotential from Dijkgraaf-Vafa matrix model, JHEP 03 (2010) 046 [arXiv:0912.2988] [SPIRES].

[52] P. Sulkowski, Matrix models for $\beta$-ensembles from Nekrasov partition functions, JHEP 04 (2010) 063 [arXiv:0912.5476] [SPIRES].

[53] A. Mironov, A. Morozov and A. Morozov, Conformal blocks and generalized Selberg integrals, Nucl. Phys. B 843 (2011) 534 [arXiv: 1003.5752] [SPIRES].

[54] A. Morozov and S. Shakirov, The matrix model version of AGT conjecture and CIV-DV prepotential, JHEP 08 (2010) 066 [arXiv: 1004.2917] [SPIRES].

[55] H. Awata and Y. Yamada, Five-dimensional AGT relation and the deformed beta-ensemble, Prog. Theor. Phys. 124 (2010) 227 [arXiv:1004.5122] [SPIRES].

[56] A. Alexandrov, Matrix models for random partitions, arXiv:1005.5715 [SPIRES].

[57] A. Morozov and S. Shakirov, From Brezin-Hikami to Harer-Zagier formulas for Gaussian correlators, arXiv:1007.4100 [SPIRES].

[58] H. Itoyama, T. Oota and N. Yonezawa, Massive scaling limit of beta-deformed matrix model of Selberg type, Phys. Rev. D 82 (2010) 085031 [arXiv: 1008.1861] [SPIRES].

[59] A. Brini, M. Mariño and S. Stevan, The uses of the refined matrix model recursion, arXiv:1010.1210 [SPIRES].

[60] A. Mironov, A. Morozov and S. Shakirov, On 'Dotsenko-Fateev' representation of the toric conformal blocks, J. Phys. A 44 (2011) 085401 [arXiv:1010.1734] [SPIRES].

[61] A. Mironov, A. Morozov and S. Shakirov, Brezin-Gross-Witten model as 'pure gauge' limit of Selberg integrals, JHEP 03 (2011) 102 [arXiv:1011.3481] [SPIRES].

[62] T. Eguchi and K. Maruyoshi, Penner type matrix model and Seiberg-Witten theory, JHEP 02 (2010) 022 [arXiv:0911.4797] [SPIRES].

[63] T. Eguchi and K. Maruyoshi, Seiberg-Witten theory, matrix model and AGT relation, JHEP 07 (2010) 081 [arXiv: 1006.0828] [SPIRES].

[64] K. Maruyoshi and F. Yagi, Seiberg-Witten curve via generalized matrix model, JHEP 01 (2011) 042 [arXiv: 1009.5553] [SPIRES].

[65] A. Marshakov, A. Mironov and A. Morozov, On AGT relations with surface operator insertion and stationary limit of beta-ensembles, J. Geom. Phys. 61 (2011) 1203 [arXiv:1011.4491] [SPIRES]. 
[66] M.C.N. Cheng, R. Dijkgraaf and C. Vafa, Non-perturbative topological strings and conformal blocks, arXiv: 1010.4573 [SPIRES].

[67] M. Goulian and M. Li, Correlation functions in Liouville theory, Phys. Rev. Lett. 66 (1991) 2051 [SPIRES].

[68] E.P. Verlinde and H.L. Verlinde, Multiloop calculations in covariant superstring theory, Phys. Lett. B 192 (1987) 95 [SPIRES].

[69] R. Dijkgraaf, E.P. Verlinde and H.L. Verlinde, $C=1$ conformal field theories on Riemann surfaces, Commun. Math. Phys. 115 (1988) 649 [SPIRES].

[70] E. D'Hoker and D.H. Phong, The geometry of string perturbation theory, Rev. Mod. Phys. 60 (1988) 917 [SPIRES].

[71] J. Bagger and M. Goulian, Coulomb gas representation on higher genus surfaces, Nucl. Phys. B 330 (1990) 488 [SPIRES].

[72] C. Vafa, Conformal theories and punctured surfaces, Phys. Lett. B 199 (1987) 195 [SPIRES].

[73] R. Donagi and E. Witten, Supersymmetric Yang-Mills theory and integrable systems, Nucl. Phys. B 460 (1996) 299 [hep-th/9510101] [SPIRES].

[74] N.A. Nekrasov and S.L. Shatashvili, Quantization of integrable systems and four dimensional gauge theories, arXiv:0908.4052 [SPIRES].

[75] A. Mironov and A. Morozov, Nekrasov functions and exact Bohr-Sommerfeld integrals, JHEP 04 (2010) 040 [arXiv:0910.5670] [SPIRES].

[76] A. Mironov and A. Morozov, Nekrasov functions from exact BS periods: the case of $\mathrm{SU}(N)$, J. Phys. A 43 (2010) 195401 [arXiv:0911.2396] [SPIRES].

[77] A. Popolitov, On relation between Nekrasov functions and BS periods in pure $\mathrm{SU}(N)$ case, arXiv: 1001.1407 [SPIRES].

[78] N. Nekrasov and E. Witten, The omega deformation, branes, integrability and Liouville theory, JHEP 09 (2010) 092 [arXiv: 1002.0888] [SPIRES].

[79] J. Teschner, Quantization of the Hitchin moduli spaces, Liouville theory and the geometric Langlands correspondence I, arXiv:1005.2846 [SPIRES].

[80] R. Poghossian, Deforming SW curve, JHEP 04 (2011) 033 [arXiv: 1006.4822] [SPIRES].

[81] B. Eynard and N. Orantin, Invariants of algebraic curves and topological expansion, math-ph/0702045 [SPIRES].

[82] B. Eynard and O. Marchal, Topological expansion of the Bethe ansatz and non-commutative algebraic geometry, JHEP 03 (2009) 094 [arXiv: 0809.3367] [SPIRES].

[83] L. Chekhov, B. Eynard and O. Marchal, Topological expansion of the Bethe ansatz and quantum algebraic geometry, arXiv:0911.1664 [SPIRES].

[84] L. Chekhov, Logarithmic potential beta-ensembles and Feynman graphs, arXiv:1009.5940 [SPIRES].

[85] L.O. Chekhov, B. Eynard and O. Marchal, Topological expansion of beta-ensemble model and quantum algebraic geometry in the sectorwise approach, Theor. Math. Phys. 166 (2011) 141 [arXiv: 1009.6007] [SPIRES].

[86] H.M. Farkas and I. Kra, Riemann surfaces, Springer-Verlag (1991). 